\title{
The covolume of quaternion groups on the four-dimensional hyperbolic space
}

\author{
by
}

Stefan Breulmann (Mannheim) and Volker Helmke (Münster)

Introduction. In this paper we compute in two different ways the volume of the fundamental domain for quaternion groups acting on the fourdimensional hyperbolic space. The first proof of our volume formula

$$
v(\mathcal{F})=\frac{\pi^{2}}{1080} \prod_{p \mid D}\left(p^{2}+1\right)(p-1),
$$

where $D$ denotes the discriminant of the corresponding rational quaternion algebra, is based on the Maaß-Selberg relations using Stokes' theorem as described in [EGM] and the Fourier expansion of the Eisenstein series $E_{R}^{*}$. The second proof uses the residue of the Eisenstein series $E_{R}^{*}$ for explicit computation of the volume. This method was already used in [Sa]. We draw the relevant Fourier expansion and the residue of the Eisenstein series from [GS].

It was kindly pointed out to the authors by F. Grunewald (Düsseldorf) that our volume formula can also be proved with some labour by the usual Tamagawa measure method. But in analogously defined cocompact situations the evaluation of the volume formula by this method seems still very complicated due to our lack of knowledge of certain Cartan involutions.

1. Preliminaries. Let $B$ be a definite rational quaternion algebra with discriminant $D$ and class number $h$. For $w=r+x i+y j+z i j \in B$ let $\bar{w}=r-x i-y j-z i j$ be the conjugate element. As usual we denote by $\mathrm{n}(w)=w \bar{w}, \operatorname{tr}(w)=w+\bar{w}, w_{\mathrm{r}}:=\frac{1}{2} \operatorname{tr}(w)$ and $\operatorname{Im} w:=\frac{1}{2}\left(w-w_{\mathrm{r}}\right)$ the norm, trace and the real and imaginary part, respectively, of an element $w \in B$.

For $A \subset B$ let $A^{(0)}:=\{a \in A: \operatorname{tr}(a)=0\}$ be the subset of all quaternions in $A$ with trace zero.

By $\mathbb{H}=B \otimes \mathbb{R}$ we denote the Hamilton quaternions. Let

$$
\mathcal{H}=\{\mathrm{P} \in \mathbb{H}: \operatorname{tr}(\mathrm{P})>0\}
$$


be the four-dimensional hyperbolic space, where we identify $\mathcal{H}=] 0, \infty[\times$ $\mathbb{R}^{3} \subset \mathbb{R}^{4}$. We always write $\mathrm{P}=(r, x, y, z)$ for $\mathrm{P} \in \mathcal{H}$ and $\mathrm{P}_{\mathrm{r}}:=r$.

The associate volume measure resp. volume form is given by

$$
d v=\frac{d r d x d y d z}{r^{4}} \quad \text { resp. } \quad d v=\frac{d r \wedge d x \wedge d y \wedge d z}{r^{4}}
$$

and the corresponding Laplace-Beltrami operator is

$$
\Delta=r^{2}\left(\frac{\partial^{2}}{\partial r^{2}}+\frac{\partial^{2}}{\partial x^{2}}+\frac{\partial^{2}}{\partial y^{2}}+\frac{\partial^{2}}{\partial z^{2}}\right)-2 r \frac{\partial}{\partial r} .
$$

Let $\operatorname{MSp}(1 ; \mathbb{H})$ (in the notation of $[\mathrm{Kr}]$, p. 316) be the modified symplectic group of degree 1 over $\mathbb{H}$, i.e.,

$$
\operatorname{MSp}(1 ; \mathbb{H})=\left\{M \in \operatorname{Mat}(2 ; \mathbb{H}): \bar{M}^{t}\left(\begin{array}{cc}
0 & 1 \\
1 & 0
\end{array}\right) M=\left(\begin{array}{cc}
0 & 1 \\
1 & 0
\end{array}\right)\right\}
$$

It is generated by the elements

$$
\left(\begin{array}{ll}
1 & a \\
0 & 1
\end{array}\right) \quad(a \in \mathbb{H}, \operatorname{tr}(a)=0), \quad\left(\begin{array}{ll}
0 & 1 \\
1 & 0
\end{array}\right), \quad\left(\begin{array}{cc}
a & 0 \\
0 & \frac{a}{n(a)}
\end{array}\right)(a \neq 0) .
$$

$\operatorname{MSp}(1 ; \mathbb{H})$ operates on $\mathcal{H}$ in the usual way $\mathrm{P} \mapsto M \mathrm{P}:=(a \mathrm{P}+b)(c \mathrm{P}+d)^{-1}$ $\left(M=\left(\begin{array}{ll}a & b \\ c & d\end{array}\right) \in \operatorname{MSp}(1 ; \mathbb{H})\right)$. The hyperbolic volume measure $d v$ is $\operatorname{MSp}(1 ; \mathbb{H})$ invariant and for $f \in C^{2}(\mathcal{H})$ and $M \in \operatorname{MSp}(1 ; \mathbb{H})$ we have

$$
\Delta(f \circ M)=(\Delta f) \circ M \text {. }
$$

Let $G$ be the group

$$
G:=\left\{M \in \operatorname{Mat}(2 ; B): \bar{M}^{t}\left(\begin{array}{cc}
0 & 1 \\
1 & 0
\end{array}\right) M=\left(\begin{array}{cc}
0 & 1 \\
1 & 0
\end{array}\right)\right\} .
$$

For a maximal order $R$ in $B$ we define $\Gamma_{R}:=\mathrm{GL}(2 ; R) \cap G$.

An element $M \in G$ is called parabolic if there exist $\gamma \in G$ and $0 \neq b \in$ $B^{(0)}$ with $\gamma^{-1} M \gamma= \pm\left(\begin{array}{ll}1 & b \\ 0 & 1\end{array}\right)$, and an element $\sigma \in \mathbb{H} \cup\{\infty\}$ is called a cusp of $\Gamma_{R}$ if $\sigma$ is fixed by some parabolic element of $\Gamma_{R}$. For a cusp $\sigma \in \mathbb{H} \cup\{\infty\}$ we denote by $\left(\Gamma_{R}\right)_{\sigma}$ the stabilizer subgroup of $\sigma$ in $\Gamma_{R}$.

Since

$$
\left(\Gamma_{R}\right)_{\infty}^{\prime}:=\left\{M \in\left(\Gamma_{R}\right)_{\infty}: \operatorname{trace} M= \pm 2\right\}=\left\{ \pm\left(\begin{array}{ll}
1 & b \\
0 & 1
\end{array}\right): b \in R^{(0)}\right\}
$$

we obtain

$$
\left[\left(\Gamma_{R}\right)_{\infty}:\left(\Gamma_{R}\right)_{\infty}^{\prime}\right]=\left|R^{\times}\right| / 2 .
$$

It is known that the set of cusps of $\Gamma_{R}$ equals $B^{(0)} \cup\{\infty\}$, and from that we can give the explicit correspondence between the classes of $\Gamma_{R}$-inequivalent cusps and the classes of left $R$-ideals in $B$ :

If we denote by $\sigma_{1}, \ldots, \sigma_{n}$ a maximal system of representatives of $\Gamma_{R^{-}}$ inequivalent cusps, the ideals $I_{i}:=R a_{i}+R c_{i}, i=1, \ldots, n\left(\sigma_{i}=a_{i} c_{i}^{-1}\right.$ 
$\left.\left(a_{i}, c_{i} \in B\right)\right)$ are a complete system of representatives of classes of left $R$ ideals, in particular we have $n=h$. We always choose the ideals $I_{i}$ such that $\mathrm{n}\left(I_{i}\right)=\mathbb{Z}$ and hence $I_{i}^{-1}=\bar{I}_{i}$, furthermore we denote by $R_{i}$ the right order of $I_{i}$.

We define the Eisenstein series for the cusp $\sigma_{i}=\gamma_{i} \infty\left(\gamma_{i} \in G\right)$ by the formula

$$
\text { (3) } \quad E_{i}(\mathrm{P}, s):=\sum_{\gamma \in\left(\Gamma_{R}\right)_{\sigma_{i}} \backslash \Gamma_{R}}\left(\gamma_{i}^{-1} \gamma \mathrm{P}\right)_{\mathrm{r}}^{s}=r^{s} \sum_{\begin{array}{c}
(a, c) \in\left(I_{i} \times I_{i}\right) / R_{i}^{\times} \\
R a+R c=I_{i} \\
\operatorname{tr}(a \bar{c})=0
\end{array}} \mathrm{n}(\bar{a} \mathrm{P}+\bar{c})^{-s} \text {, }
$$

and the modified Eisenstein series by

$$
\widetilde{E}_{i}(\mathrm{P}, s):=r^{s} \sum_{\substack{0,0) \neq(a, c) \in I_{i} \times I_{i} \\ \operatorname{tr}(a \bar{c})=0}} \mathrm{n}(\bar{a} \mathrm{P}+\bar{c})^{-s} .
$$

Both series converge for $\operatorname{Re} s>3$ uniformly on compact sets and are $\Gamma_{R^{-}}$ invariant functions since

$$
\widetilde{E}_{j}(\mathrm{P}, s)=\sum_{i=1}^{h} Z\left(I_{i j}, s\right) E_{i}(\mathrm{P}, s), \quad Z\left(I_{i j}, s\right)=\sum_{0 \neq x \in I_{i j}} \mathrm{n}(x)^{-s},
$$

with $I_{j i}:=I_{j}^{-1} I_{i}=\bar{I}_{j} I_{i}$.

The ideals $I_{j i}, i=1, \ldots, h$, constitute a complete system of representatives of the classes of left $R_{j}$-ideals, the ideal $I_{j i}$ is associated with the cusp $\sigma_{j i}:=\gamma_{j}^{-1} \sigma_{i}$ and we obtain, with an appropriate choice of $\gamma_{j}$,

$$
\Gamma_{R_{j}}=\gamma_{j}^{-1} \Gamma_{R} \gamma_{j}
$$

We define the Eisenstein series $E_{j i}^{(j)}(\mathrm{P}, s)$ and $\widetilde{E}_{j i}^{(j)}(\mathrm{P}, s)$ for the cusp $\sigma_{j i}$ as above, replacing ( $j$ fixed) $R$ by $R_{j}, I_{i}$ by $I_{j i}$ and $\sigma_{i}$ by $\sigma_{j i}$.

The correspondence between these series is given by

$$
E_{j i}^{(j)}(\mathrm{P}, s)=E_{i}\left(\gamma_{j} \mathrm{P}, s\right), \quad \widetilde{E}_{j i}^{(j)}(\mathrm{P}, s)=\widetilde{E}_{i}\left(\gamma_{j} \mathrm{P}, s\right) .
$$

Computing the Fourier coefficients of these Eisenstein series it turns out that one obtains relatively simple expressions if one forms weighted means of the Eisenstein series. The series

$$
\widetilde{E}_{R_{j}}(\mathrm{P}, s):=\sum_{i=1}^{h} \frac{1}{\left|R_{i}^{\times}\right|} \widetilde{E}_{j i}^{(j)}(\mathrm{P}, s) \quad(j=1, \ldots, h)
$$

are called weighted Eisenstein series. To get a functional equation, we multiply by an additional factor and define

$$
E_{R_{j}}^{*}(\mathrm{P}, s):=F(s) \widetilde{E}_{R_{j}}(\mathrm{P}, s) .
$$


Here $F(s)$ is defined by

$$
F(s):=\pi^{-3 s / 2+1} D^{s} \Gamma(s / 2) \Gamma(s) \zeta(2 s-2) \zeta(s-1)^{-1} \prod_{p \mid D}\left(1+p^{1-s}\right),
$$

where $\zeta$ denotes the Riemann zeta function.

The series $E_{R_{j}}^{*}(\mathrm{P}, s)$ are obviously $\Gamma_{R_{j}}$-invariant functions and satisfy the differential equation

$$
-\Delta E_{R_{j}}^{*}(\mathrm{P}, s)=s(3-s) E_{R_{j}}^{*}(\mathrm{P}, s) .
$$

We draw from [GS] the Fourier expansion of the series $E_{R_{j}}^{*}(\mathrm{P}, s)$ at infinity. This yields the Fourier expansion of $E_{R}^{*}(\mathrm{P}, s)$ at every cusp $\sigma_{j}$ (by (6)).

Theorem 1.1. The Eisenstein series $E_{R_{j}}^{*}(\mathrm{P}, s)(j=1, \ldots, h)$ have the Fourier expansions

$$
\begin{aligned}
& E_{R_{j}}^{*}(\mathrm{P}, s) \\
& =r^{s} f_{0}^{(D)}(s)+r^{3-s} f_{0}^{(D)}(3-s) \\
& \quad+4 \sqrt{\pi} \sum_{\substack{u \in R_{j}^{(0)} \\
u \neq 0}} \mathrm{n}(2 u)^{-1 / 4} \sum_{\substack{t \in \mathbb{N} \\
(t, D)=1 \\
u \in t R_{j}^{(0)}}} \sum_{\substack{D \\
q(2 u)}} t l L^{*}\left(s-1,-\frac{\mathrm{n}(2 u) D^{2}}{t^{2} l^{2}}\right) \\
& \quad \times r^{3 / 2} K_{s-3 / 2}(2 \pi r \sqrt{\mathrm{n}(2 u)}) \exp (2 \pi i \operatorname{tr}(\bar{u} \mathrm{P})) .
\end{aligned}
$$

Here the following notations are applied:

$$
f_{0}^{(D)}(s):=(s-1) D^{s} \prod_{p \mid D}\left(1-p^{2-2 s}\right) \zeta^{*}(s) \zeta^{*}(2 s-2)
$$

with the customary abbreviation

$$
\zeta^{*}(s):=\pi^{-s / 2} \Gamma(s / 2) \zeta(s) .
$$

Moreover, $\widehat{R_{j}^{(0)}}:=\left\{w \in B^{(0)}: \operatorname{tr}\left(w R_{j}^{(0)}\right) \subset \mathbb{Z}\right\}$ is the dual lattice of $R_{j}^{(0)}$ and $q(2 u)$ denotes the denominator of $\mathrm{n}(2 u)$. The function $L^{*}(s, \Delta)$ is defined by

$$
L^{*}(s, \Delta):= \begin{cases}\pi^{-s / 2} \Gamma(s / 2) \Delta^{s / 2} L(s, \Delta) & \text { for } \Delta>0 \\ \pi^{-s / 2} \Gamma((s+1) / 2)|\Delta|^{s / 2} L(s, \Delta) & \text { for } \Delta<0\end{cases}
$$

where we denote by $L(s, \Delta)$ the Cohen-Zagier L-function ([Za], p. 110), and $K_{s}$ is the modified Bessel function

$$
K_{s}(w)=\frac{1}{2} \int_{0}^{\infty} \exp \left(-\frac{w}{2}\left(t+t^{-1}\right)\right) t^{s-1} d t .
$$


Corollary 1.2. The Eisenstein series $E_{R_{j}}^{*}(\mathrm{P}, s)(j=1, \ldots, h)$ have meromorphic continuations to the whole complex plane and satisfy the functional equation

$$
E_{R_{j}}^{*}(\mathrm{P}, s)=E_{R_{j}}^{*}(\mathrm{P}, 3-s) .
$$

They are holomorphic functions except for simple poles at $s=0,3$ with the residue

$$
\operatorname{res}\left(E_{R_{j}}^{*}(\mathrm{P}, s), s=3\right)=\frac{\zeta(3)}{2 \pi} \prod_{p \mid D}\left(p^{2}-1\right)
$$

2. The volume of the fundamental domain. In this section we compute in two different ways the volume of a fundamental domain $\mathcal{F}$ for the operation of $\Gamma_{R}$ on $\mathcal{H}$. The first proof is based on the Maaß-Selberg relations, a method which was already described in [EGM]. The second proof follows the line of argument of [Sa].

2.1. First proof. For $g \in C^{1}(\mathcal{H})$ we define the differential form $\omega_{g}$ by

$\omega_{g}:=g_{r} \frac{d x \wedge d y \wedge d z}{r^{2}}-g_{x} \frac{d y \wedge d z \wedge d r}{r^{2}}+g_{y} \frac{d z \wedge d r \wedge d x}{r^{2}}-g_{z} \frac{d r \wedge d x \wedge d y}{r^{2}}$.

Let $\omega=\omega_{g}, T \in G, \mathrm{P}=(r, x, y, z) \in \mathcal{H}, \mathrm{P}^{\prime}=T \mathrm{P}=\left(r^{\prime}, x^{\prime}, y^{\prime}, z^{\prime}\right)$ and $\omega^{\prime}$ be the differential form with $\mathrm{P}$ replaced by $\mathrm{P}^{\prime}$. Simple calculations by means of the generators (1) show that

$$
\omega=\omega^{\prime} .
$$

For $g, h \in C^{1}(\mathcal{H})$ let $\omega_{(g, h)}:=\omega_{g} h$. Trivial calculations show that

$$
d \omega_{(g, h)}=\left((\Delta g) h+r^{2}(\operatorname{grad} g)^{t} \operatorname{grad} h\right) d v .
$$

Hence

$$
d \omega_{(g, h)}-d \omega_{(h, g)}=((\Delta g) h-(\Delta h) g) d v .
$$

Now consider a Poincaré normal polyhedron $\mathcal{F}$ for the operation of the group $\Gamma_{R}$ on $\mathcal{H}$. The boundary of $\mathcal{F}$ is contained in finitely many threedimensional planes (cf. [Gr], p. 215, [Wi], p. 866 ff.) and the "sides" of $\mathcal{F}$ are pairwise $\Gamma_{R}$-equivalent.

Similarly to [Ro], p. 288, we subdivide $\mathcal{F}$ in a relatively compact part and finitely many cusp sectors. For preparation we need an analogue of Shimizu's lemma.

Lemma 2.1. For $Y \geq 1$ two points $\mathrm{P}, \mathrm{P}^{\prime} \in \mathcal{H}$ with $\mathrm{P}_{\mathrm{r}}, \mathrm{P}_{\mathrm{r}}^{\prime}>Y$ are equivalent with respect to $\gamma_{j}^{-1} \Gamma_{R} \gamma_{j}=\Gamma_{R_{j}}$ if and only if they are equivalent with respect to $\left(\Gamma_{R_{j}}\right)_{\infty}$. 
Proof. Let $\mathcal{H}_{Y}:=\left\{\mathrm{P} \in \mathcal{H}: \mathrm{P}_{\mathrm{r}}>Y\right\}$. For $M \in \Gamma_{R_{j}} \backslash\left(\Gamma_{R_{j}}\right)_{\infty}, M=$ $\left(\begin{array}{ll}* & * \\ c & d\end{array}\right)$, i.e. $c \neq 0$ and $\mathrm{P} \in \mathcal{H}_{Y}$ we have

$$
(M \mathrm{P})_{\mathrm{r}}=\frac{\mathrm{P}_{\mathrm{r}}}{\mathrm{n}(c \mathrm{P}+d)}=\frac{\mathrm{P}_{\mathrm{r}}}{\mathrm{n}(c) \mathrm{n}\left(\mathrm{P}+c^{-1} d\right)} \leq \frac{\mathrm{P}_{\mathrm{r}}}{\mathrm{n}(c) \mathrm{P}_{\mathrm{r}}^{2}}<1 \leq Y,
$$

since $\operatorname{tr}\left(c^{-1} d\right)=0$ implies $\mathrm{n}\left(\mathrm{P}+c^{-1} d\right) \geq \mathrm{P}_{\mathrm{r}}^{2}$ and from $c \in R_{j}$ we conclude $\mathrm{n}(c) \geq 1$. Hence $\mathcal{H}_{Y} \cap M \mathcal{H}_{Y}=\emptyset$.

Applying Lemma 2.1 and observing that $\gamma_{j}^{-1} \mathcal{F}$ is a fundamental domain for $\Gamma_{R_{j}}$ we conclude that for a suitable choice of $\mathcal{F}$ there is a fundamental domain $\mathcal{P}_{j}$ for $\left(\Gamma_{R_{j}}\right)_{\infty}$ such that

$$
\left\{\mathrm{P} \in \mathcal{P}_{j}: \mathrm{P}_{\mathrm{r}}>Y\right\}=(] Y, \infty[\times \operatorname{Im} \mathcal{H}) \cap \gamma_{j}^{-1} \mathcal{F} .
$$

Let $\mathcal{S}_{j}$ be a cusp sector "at height" $Y$ at the cusp $\sigma_{j}$, i.e.

$$
\mathcal{S}_{j}=\gamma_{j}\left\{\mathrm{P} \in \mathcal{P}_{j}: \mathrm{P}_{\mathrm{r}}>Y\right\} \subset \mathcal{F}
$$

and

$$
\left.\left.\mathcal{F}^{Y}:=\mathcal{F} \cap\left(\bigcap_{j=1}^{h} \gamma_{j}(] 0, Y\right] \times \operatorname{Im} \mathcal{H}\right)\right)
$$

the fundamental domain "cut off at height $Y$ ". Similar considerations to the above lemma show that the cusp sectors are pairwise disjoint if $Y \geq 1$.

We now choose

$$
\begin{aligned}
& g: \mathcal{H} \rightarrow \mathbb{C}, \quad \mathrm{P} \mapsto E_{R}^{*}(\mathrm{P}, s), \\
& h: \mathcal{H} \rightarrow \mathbb{C}, \quad \mathrm{P} \mapsto E_{R}^{*}(\mathrm{P}, t)
\end{aligned}
$$

for fixed $s, t>3, s \neq t$, and let $Y \geq 1$. For $j \in\{1, \ldots, h\}, \mathrm{P}=\gamma_{j} \mathrm{P}^{\prime} \in \mathcal{S}_{j}$ with $\mathrm{P}^{\prime} \in \mathcal{P}_{j}$ and $\mathrm{P}_{\mathrm{r}}^{\prime}>Y$ the functions $g$ and $h$ have Fourier expansions at the cusp $\sigma_{j}$ of the form

$$
\begin{aligned}
& g(\mathrm{P})=\varphi\left(\mathrm{P}^{\prime}\right)+\psi_{j}\left(\mathrm{P}^{\prime}\right), \\
& h(\mathrm{P})=\sigma\left(\mathrm{P}^{\prime}\right)+\tau_{j}\left(\mathrm{P}^{\prime}\right),
\end{aligned}
$$

where

$$
\begin{aligned}
& \varphi\left(\mathrm{P}^{\prime}\right)=\left(r^{\prime}\right)^{s} f_{0}^{(D)}(s)+\left(r^{\prime}\right)^{3-s} f_{0}^{(D)}(3-s), \\
& \sigma\left(\mathrm{P}^{\prime}\right)=\left(r^{\prime}\right)^{t} f_{0}^{(D)}(t)+\left(r^{\prime}\right)^{3-t} f_{0}^{(D)}(3-t)
\end{aligned}
$$

and where $\psi_{j}, \tau_{j}$ denote the terms involving the higher Fourier coefficients in (9) with $\mathrm{P}$ replaced by $\mathrm{P}^{\prime}$.

We subtract the zeroth Fourier coefficients "in height $Y$ ", i.e.,

$$
\begin{aligned}
g^{Y}(\mathrm{P}) & := \begin{cases}g(\mathrm{P}) & \text { for } \mathrm{P} \in \mathcal{F}^{Y}, \\
g(\mathrm{P})-\varphi \circ \gamma_{j}^{-1}(\mathrm{P}) & \text { for } \mathrm{P} \in \mathcal{S}_{j},\end{cases} \\
\Delta g^{Y} & = \begin{cases}\Delta g & \text { in } \mathcal{F}^{Y}, \\
\Delta\left(g-\varphi \circ \gamma_{j}^{-1}\right) & \text { in } \mathcal{S}_{j},\end{cases}
\end{aligned}
$$


where in the last line we take the usual derivative. We conclude from (8) and simple calculations in the cusp sectors

$$
-\Delta g^{Y}=s(3-s) g^{Y}, \quad-\Delta h^{Y}=t(3-t) h^{Y} .
$$

We first treat the contribution of $\mathcal{F}^{Y}$.

LEMMA 2.2. With the same notations as above set $Q_{j}(Y):=\left\{\mathrm{P} \in \mathcal{P}_{j}\right.$ : $\left.\mathrm{P}_{\mathrm{r}}=Y\right\}$. Then

$$
\begin{aligned}
J(Y) & :=(t(3-t)-s(3-s)) \int_{\mathcal{F}^{Y}} g^{Y} h^{Y} d v \\
& =\sum_{j=1}^{h} \int_{Q_{j}(Y)}\left(g_{r^{\prime}} h-h_{r^{\prime}} g\right) \frac{d x^{\prime} d y^{\prime} d z^{\prime}}{\left(r^{\prime}\right)^{2}} .
\end{aligned}
$$

Pr o of. From (13) and (16) we have

$$
\begin{aligned}
\left((t(3-t)-s(3-s)) g^{Y} h^{Y}\right) d v & =\left(\left(\Delta g^{Y}\right) h^{Y}-\left(\Delta h^{Y}\right) g^{Y}\right) d v \\
& =d \omega_{\left(g^{Y}, h^{Y}\right)}-d \omega_{\left(h^{Y}, g^{Y}\right)}
\end{aligned}
$$

where these differential forms are defined only on $\mathcal{F}^{Y}$. Since further calculations only deal with $\mathcal{F}^{Y}$, we write $g$ and $h$ instead of $g^{Y}$ and $h^{Y}$.

Denoting by $\partial \mathcal{F}^{Y}$ the positively oriented boundary of $\mathcal{F}^{Y}$ and applying Stokes' theorem we obtain

$$
\int_{\mathcal{F}^{Y}}\left(d \omega_{(g, h)}-d \omega_{(h, g)}\right)=\int_{\partial \mathcal{F}^{Y}}\left(\omega_{(g, h)}-\omega_{(h, g)}\right) .
$$

The boundary of $\mathcal{F}^{Y}$ consists of pairwise equivalent parts of three-dimensional hyperbolic planes and the "cross-sections" $\gamma_{j} Q_{j}(Y)=\gamma_{j}\left\{\mathrm{P} \in \mathcal{P}_{j}\right.$ : $\left.\mathrm{P}_{\mathrm{r}}=Y\right\}$ at the cusps.

Since $\Gamma_{R}$-equivalent pairs of bounding hyperbolic planes inherit opposite orientation, the corresponding contributions to the above integral cancel because of (12) and the $\Gamma_{R}$-invariance of $g$ and $h$. Hence we obtain

$$
J(Y)=\sum_{j=1}^{h} \int_{\gamma_{j}}\left(\omega_{(g, h)}-\omega_{(h, g)}\right) .
$$

If we denote by $\omega_{(g, h)}^{\prime}$ the differential form with respect to $g$ and $h$ with $\mathrm{P}$ replaced by $\mathrm{P}^{\prime}=\gamma_{j} \mathrm{P}$ and similarly with $g$ and $h$ interchanged, (12) again implies

$$
J(Y)=\sum_{j=1}^{h} \int_{Q_{j}(Y)}\left(\omega_{(g, h)}^{\prime}-\omega_{(h, g)}^{\prime}\right)=\sum_{j=1}^{h} \int_{Q_{j}(Y)}\left(g_{r^{\prime}} h-h_{r^{\prime}} g\right) \frac{d x^{\prime} d y^{\prime} d z^{\prime}}{\left(r^{\prime}\right)^{2}} .
$$

This proves the assertion. 
We treat the cusp sectors applying a similar argument to the "cusp sectors cut off at height $X>Y$ "

$$
\mathcal{S}_{j}^{X}=\gamma_{j}\left\{\mathrm{P} \in \mathcal{P}_{j}: Y<\mathrm{P}_{\mathrm{r}} \leq X\right\}
$$

and letting $X \rightarrow \infty$. We only note the result.

Lemma 2.3. For an arbitrary cusp sector $\mathcal{S}_{j}$ we have

$$
\begin{aligned}
& (t(3-t)-s(3-s)) \int_{\mathcal{S}_{j}} g^{Y} h^{Y} d v \\
& =-\int_{Q_{j}(Y)}\left(\left(\frac{\partial}{\partial r^{\prime}} \psi_{j}\left(\mathrm{P}^{\prime}\right)\right) \tau_{j}\left(\mathrm{P}^{\prime}\right)-\left(\frac{\partial}{\partial r^{\prime}} \tau_{j}\left(\mathrm{P}^{\prime}\right)\right) \psi_{j}\left(\mathrm{P}^{\prime}\right)\right) \frac{d x^{\prime} d y^{\prime} d z^{\prime}}{\left(r^{\prime}\right)^{2}} .
\end{aligned}
$$

THEOREM 2.4. We have the equality

$$
\begin{aligned}
(t(3-t)-s(3-s)) & \int_{\mathcal{F}} g^{Y} h^{Y} d v \\
& =\sum_{j=1}^{h} \int_{Q_{j}(Y)}\left(\left(\frac{\partial}{\partial r^{\prime}} \varphi\right) \sigma-\left(\frac{\partial}{\partial r^{\prime}} \sigma\right) \varphi\right) \frac{d x^{\prime} d y^{\prime} d z^{\prime}}{\left(r^{\prime}\right)^{2}} .
\end{aligned}
$$

Proof. We decompose $\mathcal{F}=\mathcal{F}^{Y}+\sum_{j=1}^{h} \mathcal{S}_{j}$ and add (17) and (18). Note that the integrals over the sets $Q_{j}(Y)$ in (17) with the higher Fourier coefficients from (14) vanish because of their periodicity.

Now we can compute the volume of the fundamental domain explicitly.

THEOREM 2.5. The volume of the fundamental domain for the group $\Gamma_{R}$ is given by the equation

$$
v(\mathcal{F})=\frac{\pi^{2}}{1080} \prod_{p \mid D}\left(p^{2}+1\right)(p-1) .
$$

Proof. We compute the integrand on the right hand side of (19) by means of (15). For simplification we write $f$ instead of $f_{0}^{(D)}$ and $r$ instead of $r^{\prime}$. This gives

$$
\begin{aligned}
& \sum_{j=1}^{h} \int_{Q_{j}(Y)}\left(\left(\frac{\partial}{\partial r} \varphi\right) \sigma-\left(\frac{\partial}{\partial r} \sigma\right) \varphi\right) \frac{d x d y d z}{r^{2}} \\
& =\left(f(s) f(t) Y^{s+t-3}(s-t)+f(3-s) f(t) Y^{t-s}(3-s-t)\right. \\
& \left.\quad+f(s) f(3-t) Y^{s-t}(s+t-3)+f(3-s) f(3-t) Y^{3-s-t}(t-s)\right) \\
& \quad \times \sum_{j=1}^{h} \lambda^{3}\left(\operatorname{Im} Q_{j}(Y)\right),
\end{aligned}
$$


where $\lambda^{3}$ denotes the Lebesgue measure on $B^{(0)} \otimes \mathbb{R}=\mathbb{R}^{3}$. From (2) and $\lambda^{3}\left(B^{(0)} \otimes \mathbb{R} / R_{j}^{(0)}\right)=D / 2([\mathrm{GS}]$, p. 247$)$ we conclude that

$$
\lambda^{3}\left(\operatorname{Im} Q_{j}(Y)\right)=D /\left|R_{j}^{\times}\right| .
$$

For further simplification we set

$$
\Theta:=\sum_{i=1}^{h} \frac{D}{\left|R_{i}^{\times}\right|}=\frac{D}{24} \prod_{p \mid D}(p-1)
$$

(cf. [De], p. 134).

We now substitute $s=3+2 a, t=3+a$ with $a>0$, multiply both sides of (19) with $2 a^{2} /\left(3 a^{2}+3 a\right)$ and conclude from (20)-(22) that

$$
\begin{aligned}
2 a^{2} \int_{\mathcal{F}} g^{Y} h^{Y} d v= & \left(f(3+2 a) f(3+a) \frac{2 a^{2}}{3 a+3} Y^{3+3 a}\right. \\
& +(-2 a) f(-2 a) f(3+a) Y^{-a} \\
& -2(-a) f(-a) f(3+2 a) Y^{a} \\
& \left.+(-2 a) f(-2 a)(-a) f(-a) \frac{-1}{3 a+3} Y^{-3-3 a}\right) \Theta .
\end{aligned}
$$

The right side of this equation converges as $a \rightarrow 0+$ to

$$
\begin{aligned}
(0+f(3) \operatorname{res}(f ; 0)-2 f(3) \operatorname{res}(f ; 0)+\mathcal{O} & \left.\left(Y^{-3}\right)\right) \Theta \\
& =-f(3) \operatorname{res}(f ; 0) \Theta+\mathcal{O}\left(Y^{-3}\right) .
\end{aligned}
$$

For the same reason as in [EGM], p. 211, we obtain from (23), letting $a \rightarrow$ $0+$,

$$
v\left(\mathcal{F}^{Y}\right)=\frac{-f(3) \operatorname{res}(f ; 0) \Theta}{\left(\operatorname{res}\left(E_{R}^{*}(\mathrm{P}, s), s=3\right)\right)^{2}}+\mathcal{O}\left(Y^{-3}\right)
$$

and therefore, letting $Y \rightarrow \infty$,

$$
\begin{aligned}
v(\mathcal{F}) & =\frac{-f(3) \operatorname{res}(f ; 0) \Theta}{\left(\operatorname{res}\left(E_{R}^{*}(\mathrm{P}, s), s=3\right)\right)^{2}} \\
& =D^{4} \frac{\pi^{2}}{45} \prod_{p \mid D} \frac{1-p^{-4}}{p^{2}-1} \sum_{j=1}^{h} \frac{1}{\left|R_{j}^{\times}\right|} \quad(\text { see }(10),(11)) \\
& =\frac{\pi^{2}}{1080} \prod_{p \mid D}\left(p^{2}+1\right)(p-1) .
\end{aligned}
$$


2.2. Second proof

THEOREM 2.6. With the function $f_{0}^{(D)}$ from (10) we have for $\operatorname{Re} s>3$

$$
E_{R}^{*}(\mathrm{P}, s)=f_{0}^{(D)}(s) \sum_{j=1}^{h} \frac{2}{\left|R_{j}^{\times}\right|} \sum_{\gamma \in\left(\Gamma_{R_{j}}\right)_{\infty}^{\prime} \backslash \Gamma_{R_{j}}}\left(\gamma \gamma_{j}^{-1} \mathrm{P}\right)_{\mathrm{r}}^{s} .
$$

Pro of. Since

$$
\sum_{i=1}^{h} \frac{Z\left(I_{j i}, s\right)}{\left|R_{i}^{\times}\right|}=\zeta(s) \zeta(s-1) \prod_{p \mid D}\left(1-p^{1-s}\right)
$$

(cf. [GS], p. 255), we derive from (4) and (7) that

$$
E_{R}^{*}(\mathrm{P}, s)=f_{0}^{(D)}(s) \sum_{j=1}^{h} E_{j}(\mathrm{P}, s) .
$$

To prove the assertion, we transform the Eisenstein series $E_{j}(\mathrm{P}, s)$ in (3). From (2) and (5) we obtain

$$
E_{j}(\mathrm{P}, s)=\frac{2}{\left|R_{j}^{\times}\right|} \sum_{\gamma \in\left(\Gamma_{R_{j}}\right)_{\infty}^{\prime} \backslash \Gamma_{R_{j}}}\left(\gamma \gamma_{j}^{-1} \mathrm{P}\right)_{\mathrm{r}}^{s},
$$

which gives the desired result.

Let $\mathcal{F}$ be a Poincaré normal polyhedron for the action of $\Gamma_{R}$ on $\mathcal{H}$. Without loss of generality we may assume that $\mathcal{F}$ contains a full cusp sector for $\Gamma_{R}$ at infinity.

$\Gamma_{R}$ contains the hyperbolic rotations of the form $\gamma_{a}:=\left(\begin{array}{cc}a & 0 \\ 0 & a\end{array}\right) \in\left(\Gamma_{R}\right)_{\infty}$ with $a \in R^{\times}$. We now consider the images $\gamma_{a} \mathcal{F}$ and see that the union

$$
\mathcal{P}:=\bigcup_{a \in R^{\times} /\{ \pm 1\}} \gamma_{a} \mathcal{F}
$$

yields a set which we can assume to have the following shape (note the formula $\left(\left(\begin{array}{cc}1 & w \\ 0 & 1\end{array}\right) \gamma_{j}^{-1} \mathrm{P}\right)_{\mathrm{r}}=\left(\gamma_{j}^{-1} \mathrm{P}\right)_{\mathrm{r}}$ for all $\left.w \in R_{j}^{(0)}\right)$ :

The imaginary coordinates of the points in $\mathcal{P}$ with sufficiently large real part run through a fundamental parallelepiped $F$ for the lattice $R^{(0)}$. The real coordinates run through a set (depending on the imaginary part $\mathrm{P}-\mathrm{P}_{\mathrm{r}}$ of the points)

$$
] A\left(\mathrm{P}-\mathrm{P}_{\mathrm{r}}\right), \infty\left[\quad \text { with } A\left(\mathrm{P}-\mathrm{P}_{\mathrm{r}}\right) \geq 0 .\right.
$$

Here we have $A\left(\mathrm{P}-\mathrm{P}_{\mathrm{r}}\right)=0$ if and only if $\mathrm{P}-\mathrm{P}_{\mathrm{r}}$ is a cusp of $\Gamma_{R}$ not equivalent to $\infty$. Without loss of generality let us assume that the representatives $\sigma_{j}$ of the cusps are in $\partial \mathcal{F}$. Then the fundamental domains $\mathcal{F}_{j}:=\gamma_{j}^{-1} \mathcal{F}$, $j=1, \ldots, h$, "contain" the cusp infinity, too. Since linear-fractional transformations map hyperbolic straight lines to hyperbolic straight lines, we can 
assume for the computation of the volume that

$$
\mathcal{P}_{j}:=\bigcup_{a \in R_{j}^{\times} /\{ \pm 1\}} \gamma_{a} \mathcal{F}_{j}
$$

has the same shape as $\mathcal{P}=\mathcal{P}_{1}$ with certain $F_{j}, R_{j}^{(0)}$ and $A_{j}$ instead of $F, R^{(0)}$ and $A$, respectively. From now on we choose the fundamental domains $\mathcal{F}_{j}$ and the integration domains $\mathcal{P}_{j}$ as described above. For $a \in R^{\times}$we always denote by $\gamma_{a}$ the matrix $\left(\begin{array}{ll}a & 0 \\ 0 & a\end{array}\right)$.

Second proof of Theorem 2.5. Consider the function

$$
b(s):=\int_{\mathcal{F}}\left(E_{R}^{*}(\mathrm{P}, s)-f_{0}^{(D)}(s) \sum_{j=1}^{h}\left(\gamma_{j}^{-1} \mathrm{P}\right)_{\mathrm{r}}^{s}\right) d v(\mathrm{P}) .
$$

Since the residue of $E_{R}^{*}(\mathrm{P}, s)$ does not depend on $\mathrm{P}$, we obviously have

$$
\operatorname{res}(b(s), s=3)=v(\mathcal{F}) \operatorname{res}\left(E_{R}^{*}(\mathrm{P}, s), s=3\right) .
$$

To compute the residue of $b(s)$ in a different manner, we put $s_{n}:=3+1 / n$ and find, by Theorem 2.6,

$$
\begin{aligned}
& \operatorname{res}(b(s), s=3)=\lim _{n \rightarrow \infty}\left(s_{n}-3\right) b\left(s_{n}\right) \\
& =\lim _{n \rightarrow \infty} \frac{1}{n} \int_{\mathcal{F}} f_{0}^{(D)}\left(s_{n}\right) \sum_{j=1}^{h} \frac{2}{\left|R_{j}^{\times}\right|} \\
& \times\left(\sum_{\gamma \in\left(\Gamma_{R_{j}}\right)_{\infty}^{\prime} \backslash \Gamma_{R_{j}}}\left(\gamma \gamma_{j}^{-1} \mathrm{P}\right)_{\mathrm{r}}^{s_{n}}-\frac{\left|R_{j}^{\times}\right|}{2}\left(\gamma_{j}^{-1} \mathrm{P}\right)_{\mathrm{r}}^{s_{n}}\right) d v(\mathrm{P}) \\
& =\lim _{n \rightarrow \infty} \frac{1}{n} \int_{\mathcal{F}} f_{0}^{(D)}\left(s_{n}\right) \sum_{j=1}^{h} \frac{2}{\left|R_{j}^{\times}\right|} \sum_{\substack{\gamma \in\left(\Gamma_{R_{j}}\right)_{\infty}^{\prime} \backslash \Gamma_{R_{j}} \\
\gamma \neq \gamma_{a}, a \in R_{j}^{\times}}}\left(\gamma \gamma_{j}^{-1} \mathrm{P}\right)_{\mathrm{r}}^{s_{n}} d v(\mathrm{P}) \\
& =\lim _{n \rightarrow \infty} \frac{1}{n} \sum_{j=1}^{h} \frac{2}{\left|R_{j}^{\times}\right|} f_{0}^{(D)}\left(s_{n}\right) \sum_{\substack{\gamma \in\left(\Gamma_{R_{j}}\right)_{\infty}^{\prime} \backslash \Gamma_{R_{j}} \\
\gamma \neq \gamma_{a}, a \in R_{j}^{\times}}} \int_{\mathcal{F}_{j}} r^{s_{n}} d v(\mathrm{P}) \\
& =\lim _{n \rightarrow \infty} \frac{1}{n} \sum_{j=1}^{h} \frac{2}{\left|R_{j}^{\times}\right|} f_{0}^{(D)}\left(s_{n}\right) \int_{F_{j}}^{A_{j}(x, y, z)} \int_{0}^{1 / n-1} d r d x d y d z \\
& =f_{0}^{(D)}(3) \sum_{j=1}^{h} \frac{D}{\left|R_{j}^{\times}\right|},
\end{aligned}
$$

since $\lambda^{3}\left(F_{j}\right)=D / 2([\mathrm{GS}]$, p. 247). 
Putting together these equations we obtain from Corollary 1.2 and some well-known special values of $\Gamma$ and $\zeta$,

$$
\begin{aligned}
v(\mathcal{F}) & =\frac{f_{0}^{(D)}(3) \sum_{j=1}^{h} D /\left|R_{j}^{\times}\right|}{\operatorname{res}\left(E_{R}^{*}(\mathrm{P}, s), s=3\right)}=\frac{\pi^{2}}{45} \prod_{p \mid D}\left(p^{2}+1\right) \sum_{j=1}^{h} \frac{1}{\left|R_{j}^{\times}\right|} \\
& =\frac{\pi^{2}}{1080} \prod_{p \mid D}\left(p^{2}+1\right)(p-1) \quad(\text { see }(22)) .
\end{aligned}
$$

By way of example, in the special case of the quaternion algebra $B=$ $\left(\frac{-1,-1}{\mathbb{Q}}\right)$ and the Hurwitz order $R=\mathbb{Z} i+\mathbb{Z} j+\mathbb{Z} k+\mathbb{Z} \frac{1+i+j+k}{2}$ we have $D=2$ and obtain

$$
v(\mathcal{F})=\frac{\pi^{2}}{216}
$$

This special result can be also obtained by means of both methods from the calculations in $[\mathrm{Kr}]$.

Using the above method, we can now compute the residue of the Eisenstein series $E_{i}(\mathrm{P}, s)$ at $s=3$ without knowing the Fourier expansion explicitly. The Eisenstein series $E_{i}(\mathrm{P}, s)$ have meromorphic continuations to the half plane $\operatorname{Re} s>1$ with a simple pole at $s=3$ (cf. [GS], p. 245) and we have

$$
\operatorname{res}\left(E_{i}(\mathrm{P}, s), s=3\right)=\frac{D}{\left|R_{i}^{\times}\right| v(\mathcal{F})}
$$

\section{References}

[De] M. Deuring, Algebren, 2. Auf., Springer, Berlin, 1968.

[EGM] J. Elstrodt, F. Grunewald and J. Mennicke, Eisenstein series on threedimensional hyperbolic space and imaginary quadratic number fields, J. Reine Angew. Math. 360 (1985), 160-213.

[Gr] L. Greenberg, Finiteness theorems for Fuchsian and Kleinian groups, in: Discrete Groups and Automorphic Functions, Proc. Conf. Cambridge 1975, W. J. Harvey (ed.), Academic Press, London, 1977, 199-257.

[GS] V. A. Gritsenko and R. Schulze-Pillot, Eisenstein series on four-dimensional hyperbolic space, Acta Arith. 67 (1994), 241-268.

[Kr] A. Krieg, Eisenstein-series on real, complex and quaternionic half-spaces, Pacific J. Math. 133 (1988), 315-354.

[Ro] W. Roelcke, Das Eigenwertproblem der automorphen Formen in der hyperbolischen Ebene. II, Math. Ann. 168 (1967), 261-324.

[Sa] P. Sarnak, The arithmetic and geometry of some hyperbolic three manifolds, Acta Math. 151 (1983), 253-295.

[Wi] N. J. Wielenberg, Discrete Moebius groups: Fundamental polyhedra and convergence, Amer. J. Math. 99 (1977), 861-877. 
[Za] D. B. Zagier, Modular forms whose Fourier coefficients involve zeta functions of quadratic fields, in: Lecture Notes in Math. 627, Springer, Berlin, 1977, 105-169.

Fakultät für Mathematik und Informatik Universität Mannheim

Seminargebäude A 5

D-68131 Mannheim, Germany

E-mail: breul@hurwitz.math.uni-mannheim.de
Mathematisches Institut der Universität Münster Einsteinstraße 62

D-48149 Münster, Germany E-mail: helmke@math.uni-muenster.de 